\title{
Estilo de vida de los profesionales de enfermería del Hospital Nacional Guillermo Almenara Irigoyen, Lima
}

\author{
Lifestyle of the nursing professionals of the Hospital Nacional Guillermo Almenara Irigoyen, Lima
}

Rosa Cristina Rafael Cabanillas, Ángela Paredes Aguirre

\begin{abstract}
RESUMEN
Objetivo: Identificar el estilo de vida de los profesionales de enfermería de los servicios de medicina, cirugía y traumatología del Hospital Nacional Guillermo Almenara Irigoyen. Material y métodos: Estudio de tipo descriptivo, de diseño no experimental, de corte transversal y con un enfoque cuantitativo. Para la recolección de datos se utilizó el cuestionario de Marcos (2012) adaptado por las investigadoras que mide el estilo de vida del profesional de enfermería. Este cuestionario consta de 29 ítems que exploran seis dimensiones del estilo de vida: actividad física y deporte, alimentación, recreación, descanso, manejo del estrés y hábitos de consumo. El tipo de muestreo fue no probabilístico, eligiéndose intencionalmente a 170 profesionales de enfermería. Resultados: Del 100\% (170) de los profesionales de enfermería, el 63.5\% presenta un estilo de vida no saludable y solo el $36.5 \%$, un estilo de vida saludable. En los resultados por dimensiones: actividad física y deporte, saludable 35.9\% y no saludable 64.1\%; alimentación, saludable $37.6 \%$ y no saludable $62.4 \%$; recreación, saludable $37.1 \%$ y no saludable $62.9 \%$; descanso, saludable $31.2 \%$ y no saludable $68.8 \%$; manejo del estrés, saludable $37.1 \%$ y no saludable $62.9 \%$ y hábitos de consumo, saludable 37.1\% y no saludable 62.9\%. Conclusión: Estos resultados sugieren la necesidad de invertir en cambios, procedentes de iniciativas como reflexiones sobre el "papel del autocuidado enfermero"; así como la implementación de estrategias educativas de los programas de promoción de la salud dirigido a los profesionales de enfermería, con el fin de reducir la probabilidad de presentar comportamientos no saludables y disminuir los inminentes riesgos de morbilidad.
\end{abstract}

Palabras Clave: Estilo de vida, profesional de enfermería, hábitos saludables.

\begin{abstract}
Objective: to identify the lifestyle of the nursing professionals of the medical, surgical and traumatology services. Methods: a descriptive, non-experimental, cross-sectional study with a quantitative approach. For the data collection, the questionnaire of Marcos (2012) was adapted by the researcher who measures the nursing professional's lifestyle. This questionnaire consists of 29 items that explore six dimensions of lifestyle: physical activity and sport, food, recreation, rest, stress management and consumption habits. The type of sampling was non-probabilistic, being intentionally selected 170 nurses. Results: Of the 100\% (170) of nursing professionals, 63.5\% presented an unhealthy lifestyle and only 36.5\% a healthy lifestyle. In the results by dimensions: physical activity and sport, healthy $35.9 \%$ and unhealthy $64.1 \%$; Food, healthy $37.6 \%$ and unhealthy $62.4 \%$; Recreation, healthy $37.1 \%$ and unhealthy $62.9 \%$; Rest, healthy $31.2 \%$ and not healthy $68.8 \%$; Stress management, healthy $37.1 \%$ and unhealthy $62.9 \%$ and consumption habits, healthy $37.1 \%$ and unhealthy $62.9 \%$. Conclusions: These results suggest the need to invest in changes, from initiatives such as reflections on the "role of nursing self-care". It is important to highlight the implementation of educational strategies of the "Health Promotion and Disease Prevention Programs" aimed at nursing professionals in order to reduce the probability of presenting unhealthy behaviors and decrease the imminent risks of morbidity, with The goal of making them responsible for their decisions to change their risk behaviors, more if they are considered to be role models for both their patients and the community at large.
\end{abstract}

Keywords: Lifestyle, nursing professional, healthy habits.

${ }^{1}$ Bachiller en Enfermería, Facultad de Ciencias de la Salud, Universidad Peruana Unión, Lima, Perú.

${ }^{2}$ Doctora en Educación, Docente de la Facultad de Ciencias de la Salud, Universidad Peruana Unión, Lima, Perú. 


\section{INTRODUCCIÓN}

Los estilos de vida en la actualidad constituyen un tema de trascendencia porque, a través de su conocimiento y aplicación, permiten incrementar la proactividad de los trabajadores en el campo de la salud. Los estilos de vida que se practiquen ayudan a disminuir el riesgo de padecer de enfermedades que se pueden prevenir y, por otro lado, hará más eficientes a las personas en su vida general. La práctica de los estilos de vida saludables requiere, en gran medida, un cambio de actitud frente a los nuevos retos de la vida moderna.

El avance de la ciencia y tecnología ha traído consigo la incorporación de nuevas exigencias sociales, tales como la competitividad, la calidad y el desarrollo del proceso de globalización. A su vez, esto ha traído consigo serios retos que, al ser incorporados en los servicios de salud, ha ocasionado serias transformaciones no solo en el desempeño de los profesionales, sino también en la calidad y estilo de vida; produciendo cambios en su alimentación, actividad física y consumo de sustancias nocivas para la salud. Tal es así que es frecuente evidenciar que, debido a las exigencias y demandas sociales, el profesional de enfermería ha incrementado el consumo de una alimentación con alto contenido en grasa y carbohidratos que les puede conllevar a la obesidad y dislipidemias. Esta situación, aunada al sedentarismo y consumo excesivo de café, tabaco y otras sustancias estimulantes, puede hacer que se afecte la salud y predisponerlo a enfermedades cardiovasculares, entre otras (Barragán y De las Mercedes, 2015).

Los profesionales de la salud, al ser parte de la sociedad, están expuestos a una serie de factores de riesgos ocasionados por los estilos de vida, los cuales podrían afectar de manera directa o indirecta su salud. De ahí que es importante que los servicios de salud cuenten con un programa en el cual se considere la necesidad de proporcionar, al personal de salud, condiciones laborales que les permita contar con seguridad alimentaria que fomenten comportamientos y hábitos de nutrición saludable, la actividad física como aeróbicos, deportes, entre otras actividades, que les permita llevar una vida sana y productiva; la cual redundará en el desempeño laboral y en su calidad de vida (Ortega, 2013).

Los estilos de vida tienen gran influencia en el comportamiento del individuo en su entorno familiar, social y laboral. Entendiéndose por ello a los patrones de conducta y/o comportamiento que adopta la persona ante las actividades que realiza en su cotidianidad como hábitos y costumbres; los cuales pueden ser a mediano, corto y/o largo plazo un efecto saludable o no saludable que podría estar dado por cambios en los patrones nutricionales, sueño y descanso, recreación, actividad física y consumo de alcohol, tabaco y otros estimulantes (Mamani, 2012).

Los profesionales de enfermería algunas veces cambian sus estilos de vida, el mismo ritmo laboral al que se enfrentan altera su forma de actuar, es así cómo las jornadas laborales y los turnos de trabajo van a alterar el patrón de sueño, es decir, los horarios se ven modificados frecuentemente, así como también el número de horas que duermen; por otro lado, la nutrición también se ve afectada debido a que no cuentan con un horario de alimentación regular; al estar expuesto a estas situaciones tienen mayor riesgo de adquirir alguna enfermedad, conduciéndolo en ocasiones a automedicarse, trayendo consecuencias para su salud (Mamani, 2012).

Cabe resaltar que los estilos de vida saludables están ligados a los roles sociales, no solo por sí mismos, sino por la influencia del contexto donde se desenvuelve la vida cotidiana. La rutinización del ejercicio profesional de enfermería, ya sea en las labores asistenciales o en la docencia, progresivamente lleva a diversas formas de abandono y detrimento, tanto de la salud personal como de la calidad de vida en que se desenvuelven. Los profesionales de enfermería recomiendan a los usuarios la práctica de procesos de prevención que poco utilizan para ellos (Morales, 2008).

Quiroga (2009), en su investigación titulada “Estilos de vida y condiciones de salud de los enfermeros en el Hospital Luis Lagomaggiore”, tuvo por objetivo establecer la relación que existe entre estilo de vida y condiciones de salud del personal de enfermería, la muestra estuvo conformada por 415 enfermeros y concluyó lo siguiente: el 32\% manifestó que estuvo estresado; en relación al sueño, duerme menos de 8 horas un 93\%; el 89\% no realiza actividad física en la semana. En relación a las condiciones de salud, los datos reflejan: El 48\% consume algún tipo de bebida alcohólica, y el 61\% se considera fumador ocasional. El 55\% no tiene horario de comidas, y el resto no respeta los horarios, consumen de 2 a 3 veces comidas diarias. El 89\% tiene alguna patología y algunas enfermeras presentan más de una patología, las enfermedades más representativas son los trastornos digestivos, sobrepeso, hipertensión arterial y diabetes.

Sanabria, González \& Urrego (2007) realizaron un estudio acerca del "Estilo de vida en profesionales de salud colombianos", cuyo objetivo fue establecer la frecuencia de comportamientos saludables en una muestra de profesionales de salud. La muestra fue de 
606 profesionales de la salud, de los cuales el 17,2\% (106) fue profesional de enfermería. Se utilizó como instrumento un cuestionario adaptado sobre estilos de vida de 90 preguntas. De la población estudiada, se encontró que el 6,73\% de las enfermeras presentan un estilo de vida saludable y que el principal problema está relacionado con la actividad física y el deporte. Se concluyó que esta situación puede estar induciendo a los profesionales, además de una pobre salud en el futuro, a una actitud que no favorece la promoción de hábitos saludables en sus pacientes y la práctica de una medicina más curativa que preventiva.

Estudios como el de Mamani (2012), Marcos (2012), Galindo (2010), entre otros, demuestran que, aunque los profesionales en enfermería poseen un amplio conocimiento en promoción de estilos de vida saludables y en prevención de las enfermedades, no parece que logren interiorizarlos en su diario vivir. La situación de no interiorización de la propia promoción de estilos de vida saludables que se vislumbra en los profesionales de enfermería, a partir de estudios como estos, podría estar dando lugar a pensar en un déficit de cuidado propio dentro de los profesionales de enfermería.

Para la realización de este trabajo se escogió un tema de investigación que nace de la observación de la experiencia de la práctica profesional. El trabajo surge de observar y detectar algunas situaciones ligadas al estilo de vida y de desarrollo profesional de los profesionales de enfermería en el Hospital Nacional Guillermo Almenara Irigoyen, 2016.

Se intenta a través del desarrollo de este trabajo realizar aportes al colectivo de la profesión, tendientes al mejoramiento de la calidad de vida y de su desempeño profesional. Asimismo, este trabajo permite seleccionar y sistematizar los últimos conocimientos sobre el estilo de vida de los profesionales de enfermería sirviendo como base teórica para la comunidad científica.

\section{MATERIAL Y MÉTODOS}

Este estudio tiene un enfoque cuantitativo porque utiliza la estadística para analizar los datos, de diseño no experimental porque no pone a prueba otras variables para observar los cambios, es de corte transversal porque recolecta datos por única vez en un tiempo dado y es de tipo descriptivo porque analiza, describe y relaciona las dimensiones de la única variable de estilo de vida en los profesionales de En- fermería. El tipo de muestreo fue no probabilístico eligiéndose intencionalmente a 170 profesionales de enfermería que laboran en los servicios de medicina, cirugía y traumatología del Hospital Nacional Guillermo Almenara Irigoyen. Se eligió el tipo intencional por la facilidad de convocatoria, accesibilidad y buena disposición de brindar información de los profesionales de enfermería. Para la recolección de datos se utilizó el cuestionario de Marcos (2012) adaptado por la investigadora que mide el estilo de vida del profesional de enfermería. Este cuestionario consta de 29 ítems que exploran seis dimensiones del estilo de vida: actividad física y deporte (3 ítems); alimentación (10 ítems); recreación (2 ítems); descanso (3 ítems); manejo del estrés (5 ítems) y hábitos de consumo (6 ítems). Para el análisis estadístico se utilizó el software estadístico para Windows aplicado a las ciencias sociales SPSS22.0

\section{RESULTADOS}

Tabla 1

Estilos de vida a nivel general de los profesionales de enfermería del Hospital Nacional Guillermo Almenara Irigoyen, 2016

\begin{tabular}{lll}
\hline Estilos de vida & $\mathrm{N}$ & $\%$ \\
\hline Saludable & 62 & 36.5 \\
No saludable & 108 & 63.5 \\
Total & 170 & 100.0 \\
\hline
\end{tabular}

Tabla 2

Estilos de vida, según la dimensión Actividad Física y Deporte, de los profesionales de enfermería del Hospital Nacional Guillermo Almenara Irigoyen, 2016

\begin{tabular}{lll}
\hline $\begin{array}{l}\text { Dimensión actividad física } \\
\text { y deporte }\end{array}$ & $\mathrm{N}$ & $\%$ \\
\hline Saludable & 61 & 35.9 \\
No saludable & 109 & 64.1 \\
Total & 170 & 100.0 \\
\hline
\end{tabular}

Tabla 3

Estilos de vida, según la dimensión Alimentación, de los profesionales de enfermería del Hospital Nacional Guillermo Almenara Irigoyen, 2016

\begin{tabular}{lll}
\hline Dimensión alimentación & $\mathrm{N}$ & $\%$ \\
\hline Saludable & 64 & 37.6 \\
No saludable & 106 & 62.4 \\
Total & 170 & 100.0 \\
\hline
\end{tabular}


Tabla 4

Estilos de vida, según la dimensión Recreación, de los profesionales de enfermería del Hospital Nacional Guillermo Almenara Irigoyen, 2016

\begin{tabular}{lll}
\hline Dimensión recreación & $\mathrm{N}$ & $\%$ \\
\hline Saludable & 63 & 37.1 \\
No saludable & 107 & 62.9 \\
Total & 170 & 100.0 \\
\hline
\end{tabular}

Tabla 5

Estilos de vida, según la dimensión Descanso, de los profesionales de enfermería del Hospital Nacional Guillermo Almenara Irigoyen, 2016

\begin{tabular}{lll}
\hline Dimensión descanso & $\mathrm{N}$ & $\%$ \\
\hline Saludable & 53 & 31.2 \\
No saludable & 117 & 68.8 \\
Total & 170 & 100.0 \\
\hline
\end{tabular}

Tabla 6

Estilos de vida, según la dimensión Manejo del Estrés, de los profesionales de enfermería del Hospital Nacional Guillermo Almenara Irigoyen, 2016

\begin{tabular}{lll}
\hline Dimensión manejo del estrés & $\mathrm{n}$ & $\%$ \\
\hline Saludable & 107 & 62.9 \\
No saludable & 63 & 37.1 \\
Total & 170 & 100.0 \\
\hline
\end{tabular}

Tabla 7

Estilos de vida, según la dimensión Hábitos de Consumo, de los profesionales de enfermería del Hospital Nacional Guillermo Almenara Irigoyen, 2016

\begin{tabular}{lll}
\hline Dimensión hábitos de consumo & $\mathrm{N}$ & $\%$ \\
\hline Saludable & 63 & 37.1 \\
No saludable & 107 & 62.9 \\
Total & 170 & 100.0 \\
\hline
\end{tabular}

\section{DISCUSIÓN}

Los resultados de la investigación muestran que los estilos de vida de los profesionales evaluados presentan en general deficiencias, sobre todo en aspectos como la actividad física y el deporte. Los profesionales de enfermería que cuidan su salud y practican estilos de vida saludables tienen mayor probabilidad de recomendar y de inducir en sus pacientes hábitos igualmente saludables y de tener mayor éxito en el seguimiento de sus recomendaciones; contrario a aquellos de hábitos no saludables, que tienden a prac- ticar una medicina curativa en lugar de preventiva y que probablemente son poco escuchados por sus pacientes cuando les recomiendan comportamientos que evidentemente ellos tampoco practican (Marcos, 2012). De ahí que el tema de los estilos de vida adquiere mayor importancia cuando se trata de profesionales de la salud, pues el impacto sobre la salud pública es doble: además de impactar a un grupo en particular, los estilos de vida de dicho grupo recaen en las acciones de salud de sus pacientes, al convertirse en modelos de vida para ellos (Frank, 2006).

En relación a los estilos de vida, según la dimensión Actividad Física y Deporte, Rosales (2002) refiere que el 64\% de profesionales de enfermería no practican ejercicios físicos con regularidad y Cajaleón y Sánchez (2015) refieren que el 70\% de las enfermeras del servicio de Cirugía del HNGA nunca participan en programas de actividades de ejercicio. Ambos respaldan los resultados encontrados en la tabla 3, la cual evidencia que la mayoría (64.1\%) de los profesionales de enfermería presentan un estilo de vida no saludable, mientras que el $35.9 \%$ presenta un estilo de vida saludable.

Actualmente, los hábitos alimenticios se han convertido en inadecuados debido al aumento de la prosperidad y la urbanización, estas dietas tienden a ser más ricas en energías y en grasas (especialmente saturadas) y a tener un contenido mayor de carbohidratos complejos (Sanabria et al, 2007).

Desde el punto de vista de Cajaleon y Sánchez (2015) tener una dieta saludable es una de las cosas más importantes que se debe practicar para mantener buena salud en general. La Ley Nº636 ejercicio de la profesión de enfermería Art. 9, España, establece que el personal de enfermería debe contar con un espacio físico adecuado donde pueda ingerir sus alimentos establecidos por ley. Sin embargo, en nuestro país todavía no se garantiza una estructura adecuada de trabajo, por lo tanto, es remoto que las autoridades puedan admitir un espacio en el que el trabajador de salud pueda alimentarse.

Es importante destacar que la alimentación es un elemento esencial en la buena salud, influye la calidad de los alimentos, la cantidad de comida y los hábitos alimentarios para un bienestar del ser humano, con lo cual se obtiene una nutrición equilibrada. A nivel biológico están la edad y el crecimiento: en el adulto maduro el enlentecimiento del metabolismo hace que disminuyan las necesidades nutricionales en cantidad pero no en calidad; debe consumir una dieta balanceada, baja en grasa, condimentos y harinas. Igualmente, la actividad física influye puesto que cuanto mayor es la actividad muscular más energía se quema, ne- 
cesitando una porte suplementario de alimentos que le proporcionen la energía gastada (Herrera, 2008).

De la misma manera, la Organización Mundial de la Salud (2003) reconoce que la epidemia creciente de enfermedades crónicas, que aqueja tanto a países desarrollados como a los países en desarrollo, está relacionada con los cambios de los hábitos alimentarios y del modo de vida. De allí la necesidad de intervenir con el objetivo de generar un cambio en los hábitos y patrones de consumo alimentario.

Por estas razones, se debe tomar en cuenta, de manera importante, los resultados obtenidos en la tabla 4 donde se observa que la mayoría (62.4\%) de los profesionales de enfermería del Hospital Nacional Guillermo Almenara presentan una alimentación no saludable mientras que solo el 37.6\%, una alimentación saludable. En esa misma línea Cajaleón y Sánchez (2015), en sus resultados de estilo de vida y dimensión alimentación de las enfermeras del servicio de cirugía del HNGAI, encontraron que el 62.16\% tiene una alimentación no saludable, donde la tendencia negativa se determina en el no consumo de 6 a 8 vasos de agua al dia y no comer de acuerdo a los requerimientos nutricionales; solo el $37.84 \%$ presenta una alimentación saludable.

Por otra parte, en la tabla 5 se observa que del 100\% (170) de los profesionales de enfermería del Hospital Nacional Guillermo Almenara Irigoyen, el 62.9\% presenta un estilo de vida no saludable en la dimensión recreación y solo el 37.1\%, un estilo de vida saludable. Este resultado es compatible con el encontrado por Marcos (2012) donde se evidencia que el 67\% de los profesionales de enfermería presentan estilos de vida no saludable, en la dimensión recreación.

Según Mamani (2012) los estilos de vida tienen gran influencia en el comportamiento del individuo en su entorno familiar, social y laboral. Entendiéndose por ello a los patrones de conducta y/o comportamiento que adopta la persona ante las actividades que realiza en su cotidianidad como hábitos y costumbres; los cuales pueden ser a mediano, corto y/o largo plazo un efecto saludable o no saludable que podría estar dado por cambios en los patrones nutricionales, sueño y descanso, recreación, actividad física y consumo de alcohol, tabaco y otros estimulantes.

En la actualidad, la profesión de enfermería está consciente de la necesidad de practicar estilos de vida saludable para alcanzar calidad de vida y un buen desempeño laboral. Sin embargo, el profesional de enfermería invierte menos tiempo para el descanso, recreación, salud y familia, lo cual repercute negativamente en su desarrollo profesional (Carrillo \& Amaya, 2006).
En cuanto a los estilos de vida, según la dimensión descanso, de los profesionales de enfermería del HNGAI, se observa que la gran mayoría 68.8\% (117) presenta un estilo de vida no saludable y solo el 31.2\% (53), un estilo de vida saludable. Estos resultados son compatibles con los encontrados por Cajaleón y Sánchez (2015) los cuales muestran que el $75.68 \%$ de los profesionales de enfermería presentaban trastornos del sueño, destacando como principales factores los horarios rotativos y las guardias.

Asimismo, Quiroga (2009), en su investigación titulada "Estilos de vida y condiciones de salud de los enfermeros en el Hospital Luis Lagomaggiore”, encontró que el 93\% de los profesionales duermen menos de 8 horas, un 93\%. Estos resultados corroboran lo mencionado por Carrillo y Amaya (2006): "Durante el sueño se alcanza una reparación física y psíquica completa. Los trastornos de sueño abarcan a todos los ámbitos de la vida, tanto laboral, social como familiar; no solo para quienes la padecen, sino para quienes comparten su vida. Así, si el profesional de enfermería no descansa lo suficiente, se sentirá cansado en el área de trabajo, teniendo un bajo desempeño laboral y teniendo consecuencias negativas en su estilo y calidad de vida.

En la tabla 7 se aprecia que del 100\% (170) de los profesionales de enfermería, el 62.9\% maneja el estrés en una forma no saludable y solo el $37.1 \%$ lo hace saludablemente. De igual forma, Umaña (2005), en su investigación "Relación entre estilos de vida y condición de salud de las (os) profesionales en enfermería del Hospital de Guápiles, Costa Rica”, encontró que un alto porcentaje de las (os) profesionales presentan estilos de vida no saludables. El perfil de morbilidad de la población estudiada incluye: agotamiento físico y mental, alteraciones del estado de ánimo y del sueño, sobrecarga laboral y estrés. Concluyó que el proceso de trabajo en enfermería tiene implicancias negativas en la salud del profesional y la asociación entre elementos del estilo de vida y de la condición de salud potencia el riesgo de daño a la salud del profesional.

Asimismo, Cajaleón y Sánchez (2015), en sus resultados de estilos de vida y dimensión manejo del estrés de las enfermeras del servicio de Cirugía del HNGAI, encontraron que un $51.35 \%$ presenta un manejo del estrés no saludable, mientras que solo el $48.65 \%$ lo maneja saludablemente.

Estos resultados concuerdan con lo mencionado por Fernández (2010): "La enfermería es una profesión especialmente vocacional de ayuda y cuidados; posee altos niveles de responsabilidad, relaciones interpersonales y exigencias sociales, lo que hace que esta 
profesión sea más vulnerable al estrés laboral; circunstancias de trabajo desarrollado, muchas horas unidas al sufrimiento del paciente, ser intermediarios entre el paciente, el médico y la familia, el poco reconocimiento social y soportar exigencias de ambos lados conlleva a la acumulación de tensiones emocionales y relacionales. En enfermería, las dificultades organizativas son un marco adecuado para que pueda instaurarse el estrés laboral. La carga de trabajo, horarios, problemas administrativos, problemas de relación entre compañeros y jefes inmediatos, la toma de decisiones en situaciones de urgencia, son los mayores problemas estresantes.

A su vez, la investigación de Bello (2013) refiere que las situaciones que pueden causar estrés en los profesionales de enfermería son: la responsabilidad del trabajo, la presencia excesiva de ruidos, la iluminación defectuosa, espacio inadecuado o insuficiente para las labores a realizar, exceso de calor, ausencia de personal médico en situaciones de urgencia, ambigüedad en la asignación de las funciones de enfermería, falta de personal para atender adecuadamente el servicio, sobrecarga de trabajo, críticas constantes por parte de otros profesionales de la salud, falta de comunicación entre los compañeros de trabajo sobre los problemas del servicio, información insuficiente o inadecuada con respecto a los pacientes, rotación permanente en diferentes servicios. Tales condiciones o eventos estresantes pueden desencadenar consecuencias que inciden en el deterioro eminente de su salud, en todas las esferas biopsicosocial y, por ende, una degradación del desempeño laboral que afecta al mismo equipo de salud y también al usuario hospitalizado

Para finalizar con los resultados encontrados en la presente investigación, la tabla 8 muestra que el $62.9 \%$ de los profesionales de enfermería tienen hábitos de consumo no saludables; no obstante, solo el 37.1\% presenta hábitos de consumo saludables.

En esa misma línea, Quiroga (2009), en su investigación titulada "Estilos de vida y condiciones de salud de los enfermeros en el Hospital Luis Lagomaggiore”, encontró que el 48\% consume algún tipo de bebida alcohólica, y el $61 \%$ se considera fumador ocasional. Frente a estos resultados, se debe reflexionar en lo que menciona White (1965): "El consumo continuo de estos excitantes de los nervios provoca dolor de cabeza, insomnio, palpitaciones del corazón, indigestión, temblores y otros muchos males; porque esos excitantes consumen las fuerzas vitales. Los nervios cansados necesitan reposo y tranquilidad, en vez de estímulo y recargo de trabajo. La naturaleza necesita tiempo para recuperar las agotadas energías. Cuando sus fuerzas son aguijoneadas por el uso de estimulantes uno puede realizar mayor tarea; pero cuando el or- ganismo queda debilitado por aquel uso constante se hace más difícil despertar las energías hasta el punto deseado. Es cada vez más difícil dominar la demanda de estimulantes hasta que la voluntad queda vencida y parece que no hay poder para negarse a satisfacer un deseo tan ardiente y antinatural, que pide estimulantes cada vez más fuertes, hasta que la naturaleza, exhausta, no puede responder a su acción”.

Asimismo, Morales (2008) destaca que los profesionales de enfermería recomiendan a los usuarios la práctica de procesos de prevención que poco utilizan para ellos.

En términos generales, el autocuidado hoy más que nunca se hace imprescindible en la vida de cada ser, el autocuidado parte, entonces, de una racionalidad y de una subjetividad que atraviesa diversas esferas del ser humano. No desconoce la compleja historia de vida que acompaña a cada persona a través de la cual ha socializado sus afectos, desafectos, intereses, opiniones dentro de relaciones en donde el conflicto y la contradicción hacen parte de la cotidianeidad de la vida.

En resumen, impactar sobre el estilo de vida implica la toma de conciencia de los profesionales de enfermería sobre el compromiso y responsabilidad que se tiene en el cuidado de su propia salud y el desarrollo de nuestro potencial como seres humanos y como profesionales de enfermería que tienen una gran responsabilidad social; es por ello, que debemos involucrarnos más con los programas de promoción de estilos de vida y trabajo saludable, y que ello se replique en la población con el autocuidado y la educación con refuerzo visual constante.

Este estudio es un punto de partida para la realización de otras investigaciones, sobre los estilos de vida y hábitos de salud de los profesionales, vinculadas con las distintas instituciones prestadoras de servicios de salud de otras zonas del país, con el fin de crear programas conjuntos que lleven a una mejor calidad de vida, tanto para los usuarios como para el personal de salud, en especial para los profesionales de enfermería que tienen la responsabilidad social de velar por el cuidado.

\section{Declaración de financiamiento y de conflicto de in- tereses:}

El estudio fue financiado por los autores, quienes declaran no tener algún tipo de conflicto de interés en la investigación realizada.

\section{Correspondencia:}

Rosa Cristina Rafael Cabanillas

e-mail: rosarafaelcabanillas@gmail.com 


\section{REFERENCIAS BIBLIOGRÁFICAS}

Anguita, C. y Rodríguez, C. (2011). Valoración de los estilos de vida del personal de enfermería expuesto a turnicidad en el Centro Hospitalario de Jaén. (Tesis de licenciatura). Universidad Privada del Sur, Perú.

Atance, M. (1997). Aspectos epidemiológicos del síndrome de burnout en personal sanitario. Revista Española de Salud Pública, 7(3), 293-303.

Baggio, O. (2006). Significado de cuidado para profissionais da equipe de enfermagem. Revista de Enfermería, 8 (1), 9-16. Recuperado de http://www.fen.ufg.br/revista/ revista8_1/original_01.htm.

Barragán, L. y De las Mercedes, A. (2015). Estilos de vida saludables en personal docente, administrativo y de apoyo de la escuela de enfermería de la Universidad de Guayaquil 2015. (Tesis de licenciatura). Universidad de Guayaquil, Guayaquil, Ecuador.

Cajaleón, A y Sánchez, J. (2015). Estilo de vida de los profesionales de enfermería del Hospital Nacional Guillermo Almenara Irigoyen. (Tesis de especialidad). Universidad Peruana Unión, Lima, Perú.

Carrillo, H. y Amaya, M. (2006). Estilos de vida saludables de las enfermeras (os) docentes de la Escuela de Enfermería de la Universidad Pedagógica y Tecnológica de Colombia. (Tesis de licenciatura). Universidad Pedagógica y Tecnológica de Colombia, Colombia

Díaz, N. (2007). Estilos de vida relacionados con alimentación y salud mental, de las enfermeras del Sanatorio Nuestra Señora del Pilar. (Tesis de licenciatura). Escuela Nacional de Enfermeras de Guatemala, Facultad de Ciencias Médicas, Guatemala.

Frank, E. (2006). Her Medical Specialty is Preventive Medicina. Canada

Galindo, R. (2010). Estilos de vida del profesional asistencial de salud del hospital regional de Ayacucho y Hospital II ESSALUD Huamanga. (Tesis de licenciatura). Universidad Nacional de San Cristóbal de Huamanga, Perú.

Gálvez, C. (2008). Viva Mejor con más salud. Florida: ACES.

Gerónimo, M., Mendieta, M. y Moccia, A. (2014). Opinión acerca de las condiciones de trabajo y estilo de vida en el área de Enfermería. (Tesis de licenciatura), Universidad Nacional de Córdoba). Recuperado de http://www. enfermeria.fcm.unc.edu.ar/biblioteca/tesis/geronimo_ maria.pdf

Giboons, G. (2001). La actividad física como parte de un estilo de vida saludable. España: Elsevier

Laguado, E. y Gomez, M. (2014). Estilos de vida saludable en estudiantes de enfermería en la universidad cooperativa de Colombia. Hacia la Promoción de la Salud, 19(1), 68-83. Recuperado de http://www.redalyc. org/pdf/3091/309131703006.pdf

Mamani, B. (2012). Estilos de vida saludable que practica el profesional de Enfermería del Hospital Daniel A. Carrión (Essalud-Calana) Tacna - 2012. (Tesis de licenciatura, Universidad Nacional Jorge Basadre Grohmann. Recuperado de http://tesis.unjbg.edu. pe:8080/handle/unjbg/296

Maya, L. (2011). Estilos de vida saludables: Componente de la calidad de vida. Costa Rica: Funlibre

Marcos Montero, C. (2012). Estilos de vida y Síndrome de Burnout en profesionales de enfermería del Hospital Guillermo Almenara Irigoyen. (Tesis de licenciatura). Escuela de Enfermería Padre Luis Tezza, Universidad Ricardo Palma, Lima, Perú.

Mendoza M. (2007). Estilos de Vida de los estudiantes de la Universidad Nacional Mayor de San Marcos (Tesis de especialidad). Universidad Nacional Mayor de San Marcos, Lima, Perú.

Organización Mundial de la Salud.(2013). WorldHealthReport. Reducingriskshealthylife. Recuperado de http://www. paho.org

Organización Mundial de la Salud. (2008). World Health Report. Reducing risks promoting healthy life. Recuperado de: http://www.paho.org

Ortega, Sanchez, J. (2013). Prácticas de estilo de vida saludable en el profesional de enfermería del servicio de emergencia del Hospital Carlos Lanfranco La Hoz de Puente Piedra. (Tesis de especialidad). Universidad Nacional Mayor de San Marcos. Lima, Perú.

Pellegrino, E. (1985). The caring ethics. Alabama: University of Alabama Press.

Pérez, V. (2008). Actividad física, salud y actitudes. Valencia: Edetania.

Reyes, S. (2008). Factores que intervienen a llevar estilos de vida saludables en el personal de enfermería en los servicios de medicina y cirugía de hombres y mujeres en el Hospital San Benito, Petén. (Tesis de licenciatura). USAC - Escuela Nacional de Enfermería, Guatemala.

Rojas Cuadros, A. (2009). Estilos de vida de los profesionales de enfermería del Hospital Central FAP. Lima - 2009. (Tesis de licenciatura). Universidad Nacional Mayor de San Marcos, Lima-Perú

Rosales Chávez de Tuyuc E. (2002). Hábitos personales relacionados con alimentación, sueño y ejercicio físico de las enfermeras del Hospital Roosevelt, Guatemala. (Tesis de licenciatura). Universidad Mariano Gálvez, Guatemala.

Romero, M., Mesa, L. y Galindo, S. (2008). La calidad de vida de las(os) profesionales de enfermería y sus consecuencias en el cuidado. Avances en enfermería, 26(2), 59-70. Recuperado de http://www.index-f.com/ rae/262/r5970.php

Sanabria, González y Urrego. (2007). Estilo de vida en profesionales de salud colombianos. Revista Med, 15(2), 207 - 217. Recuperado de http://www.scielo.unal.edu. co/scielo.php?script=sci_arttext\&pid=S0121525620070 00200008\&lng=es\&nrm= 
Umaña, A. (2005). Relación entre estilos de vida y condición de salud de los profesionales en enfermería, Hospital de Guápiles. Recuperado de http://www.binasss.sa.cr/ revistas/enfermeria/v28n1/art2.pdf.
Valdivia, C. (2004). Estudio de estilos de vida en estudiantes que cursan el último año de su carrera de pregrado en la Universidad Austral de Chile de Enfermería. (Tesis de licenciatura) Universidad Austral de Chile, Chile.

Recibido: 10/11/2016

Aceptado: 01/12/2016 